\title{
On-Line Appendix Statistical Non-Significance in Empirical Economics
}

Alberto Abadie

Current version: August 30, 2019

\section{Conditioning on the Sign of the Estimated Coefficient:}

In the main text of this article we have shown that statistical significance may carry very little information in large samples. As a result, the values of other statistics should be taken into account along with significance when the null is rejected in a significance test. As discussed in Section 2, in a normal (or asymptotically normal) setting it does not take much to go back to full information (e.g., two-sided $p$-value and the sign of $\widehat{\theta}_{n}$ ). Here we consider the question of whether minimally augmenting the information on significance with the sign of $\widehat{\theta}_{n}$ results in informativeness when the null is rejected. This exercise is motivated by the possibility that the sign of the estimated coefficient is implicitly taken into account in many discussions of results from significance tests.

For concreteness, we will concentrate on the case of a positive coefficient estimate, $\widehat{\theta}_{n}>0$. That is, the limited information posterior under significance and positive $\widehat{\theta}_{n}$ conditions on the event $\sqrt{n} \widehat{\theta}_{n}>c$. The case with negative $\widehat{\theta}_{n}$ is analogous. Using similar calculations as in Section 1, we obtain:

$$
p\left(\theta \mid \sqrt{n} \widehat{\theta}_{n}>c\right)=\frac{\frac{1}{\sigma} \phi\left(\frac{\theta-\mu}{\sigma}\right) \Phi(\sqrt{n} \theta-c)}{\Phi\left(\frac{\sqrt{n} \mu-c}{\sqrt{1+n \sigma^{2}}}\right)},
$$

and

$$
p\left(\theta \mid 0<\sqrt{n} \widehat{\theta}_{n} \leq c\right)=\frac{\frac{1}{\sigma} \phi\left(\frac{\theta-\mu}{\sigma}\right)(1-\Phi(\sqrt{n} \theta-c)-\Phi(-\sqrt{n} \theta))}{1-\Phi\left(\frac{\sqrt{n} \mu-c}{\sqrt{1+n \sigma^{2}}}\right)-\Phi\left(\frac{-\sqrt{n} \mu}{\sqrt{1+n \sigma^{2}}}\right)}
$$

Figure A.1 reproduces the setting of Figure 1 but for the case when the posterior is conditional on the sign of the estimate in addition to significance. Like in Figure 1, failure to reject carries substantial information. In fact, both outcomes of the significance test carry additional information, with respect to the setting in Figure 1, which of course is explained by the additional information in the sign of $\widehat{\theta}_{n}$. 


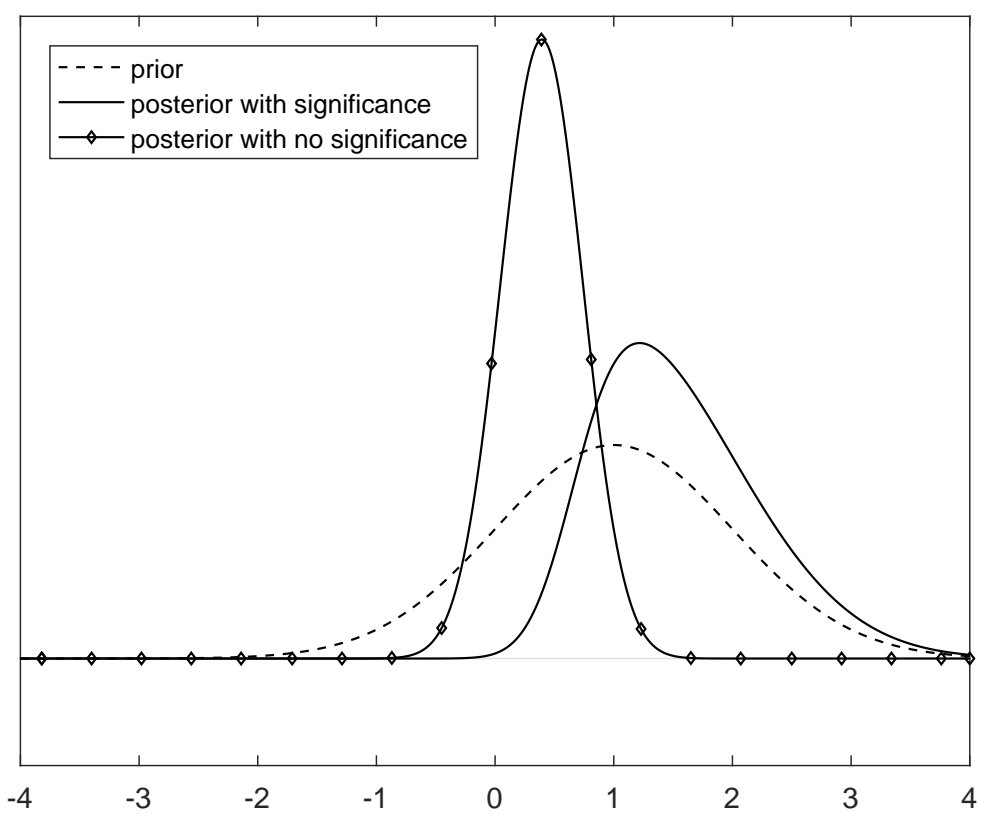

Figure A.1: Posterior Distributions Conditional on Significance and Positive Coefficient Sign

Notice that, in this case, under significance, the ratio between the posterior and the prior converges to

$$
\lim _{n \rightarrow \infty} \frac{p\left(\theta \mid \sqrt{n} \widehat{\theta}_{n}>c\right)}{p(\theta)}= \begin{cases}0 & \text { if } \theta<0 \\ \Phi(-c) / \Phi(\mu / \sigma) & \text { if } \theta=0 \\ 1 / \Phi(\mu / \sigma) & \text { if } \theta>0\end{cases}
$$

Without significance, the ratio between the posterior and the prior converges to

$$
\lim _{n \rightarrow \infty} \frac{p\left(\theta \mid 0<\sqrt{n} \widehat{\theta}_{n} \leq c\right)}{p(\theta)}= \begin{cases}0 & \text { if } \theta \neq 0 \\ \infty & \text { if } \theta=0\end{cases}
$$

That is, as $n \rightarrow \infty$ non-significance is highly informative. Under significance, the posterior of $\theta$ converges to the prior truncated at zero. As a result, in this case the informational content of significance depends on the value of $\operatorname{Pr}(\theta>0)=\Phi(\mu / \sigma)$. If this quantity is small, significance with a positive sign is highly informative. Unsurprisingly, when $\mu / \sigma$ is large (that is, in cases where there is little uncertainty about the sign of the parameter of interest), a positive sign of $\widehat{\theta}_{n}$ does not add much to the informational content of the test. Moreover, the limit of $p\left(\theta \mid \sqrt{n} \widehat{\theta}_{n}>c\right)$ cannot be more than double the value of $p(\theta)$ as long as $\mu$ is non-negative. This is relevant to many instances in economics where there are strong beliefs about the sign of the estimated coefficients (e.g., the slope of the demand 
function, or the effect of schooling on wages) and specifications reporting "wrong" signs for the coefficients of interest are rarely reported or published. ${ }^{1}$

\section{Testing an Interval Null:}

In view of the lack of informativeness of significance in large samples (under a point null), one could instead try to reinterpret significance tests as tests of the implicit null " $\theta$ is close to zero".

To accommodate this possibility, we will now concentrate on the problem of testing the null that the parameter $\theta$ is in some interval around zero. Under the null hypothesis, $\theta \in[-\delta, \delta]$, where $\delta$ is some positive number. Under the alternative hypothesis, $\theta \notin[-\delta, \delta]$. Consider the normal model of Section 2. To obtain a test of size $\alpha$ we control the supremum of the probability of Type I error:

$$
\operatorname{Pr}\left(\sqrt{n}\left|\widehat{\theta}_{n}\right|>c|| \theta \mid=\delta\right)=\Phi(\sqrt{n} \delta-c)+\Phi(-\sqrt{n} \delta-c) .
$$

Therefore, we choose $c$ such that $\Phi(\sqrt{n} \delta-c)+\Phi(-\sqrt{n} \delta-c)=\alpha$. While there is no closed-form solution for $c$, its value can be calculated numerically for any given value of $\sqrt{n} \delta$, and a very accurate approximation for large $\sqrt{n} \delta$ is given by

$$
c=\Phi^{-1}(1-\alpha)+\sqrt{n} \delta .
$$

That is, controlling size in this setting implies that the critical value has to increase with the sample size at a root- $n$ rate, with the constant given by $\delta$. In turn, this implies that the probability of rejection, $\operatorname{Pr}\left(\sqrt{n}\left|\widehat{\theta}_{n}\right|>c \mid \theta\right)=\Phi(\sqrt{n} \theta-c)+\Phi(-\sqrt{n} \theta-c)$ converges to one if $\theta \notin[-\delta, \delta]$, and converges to zero if $\theta \in(-\delta, \delta)$. As a result, the large sample posterior distributions with and without significance are truncated versions of the prior, with the prior truncated at $(-\delta, \delta)$ under significance, and at $(-\infty,-\delta) \cup(\delta, \infty)$ under no significance. If $\delta$ is large, both significance and non-significance are informative. If, however, $\delta$ is small, we go back to the setting where significance carries only local-to-zero information. Figure A.2 reports exact prior and posterior distributions for the same prior as in Figure 1, and with $\delta=\{0.5,1,2\}, \alpha=0.05$ and $n=10000$. When $\delta$ is small the large sample posterior under significance is close to the prior and the large sample posterior under no significance is close to a distribution degenerate at zero.

\section{References}

Kennedy, P. E. (2005). Oh no! I got the wrong sign! What should I do? The Journal of Economic Education 36(1), 77-92.

\footnotetext{
${ }^{1}$ The notion that empirical estimates might display "wrong" signs is widespread to the point that econometric articles and textbooks discuss this phenomenon and, in some cases, potential remedies. See, e.g., Wooldridge (2016) and Kennedy (2005).
} 

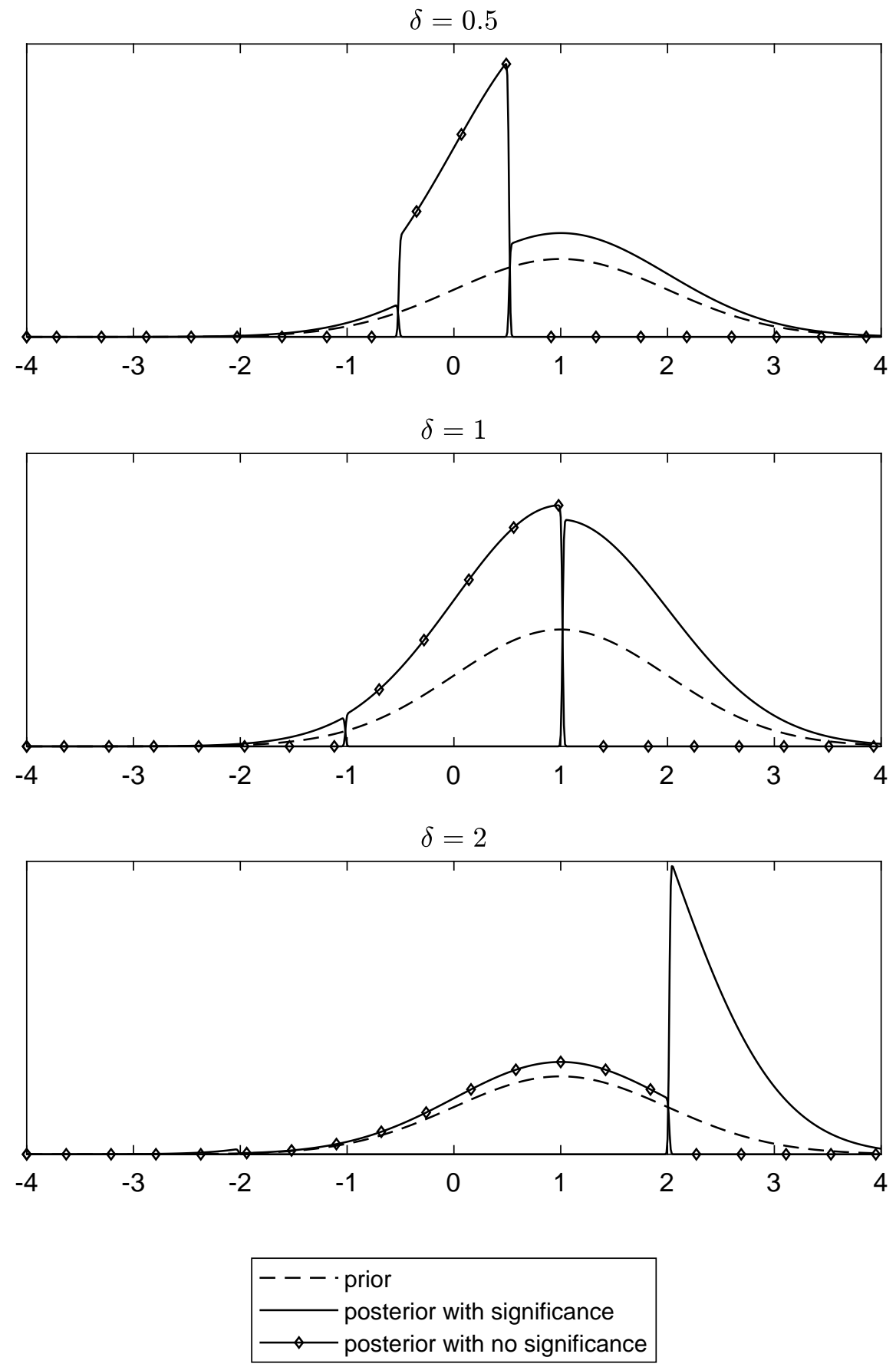

Figure A.2: Posterior After a Test of the Null $\theta \in[-\delta, \delta](n=10000, \alpha=0.05)$ 
Wooldridge, J. M. (2016). Introductory econometrics : A modern approach. Boston: Cengage Learning. 